\title{
Transport-Health Equity Outcomes from mobile phone location data - a case study
}

\author{
Grant-Muller, Susan ${ }^{\text {a }}$; Hodgson, Frances ${ }^{\text {a }}$; Harrison, Gillian ${ }^{\text {a }}$; Malleson, Nick ${ }^{\text {b }}$ \\ Redferen, Tom ${ }^{b}$ and Snowball, Rob ${ }^{c}$ \\ a Institute for Transport Studies, University of Leeds, UK, 'bchool of Geography, \\ University of Leeds, $\mathrm{UK}^{\mathrm{c}}$ Newcastle City Council, Newcastle, UK.
}

\begin{abstract}
Existing cross-sectoral models of transport-health interactions have been largely developed based on traditional (mainly aggregate) data sources e.g. travel diaries, traffic counts. Such modelling provides a 'snapshot' of the transport system at fixed locations and is prone to data related weaknesses. However dissagregate health equity consequences are needed for policy development and to direct mitigating financial resources.

This research harnesses new generation smartphone location data to collect day-to-day movement patterns of individuals via an app (with consent), including mode, route and other information i.e. Track and Trace data. A case study is presented from Newcastle City region (UK), for July 2017 onwards, (circa 2000 individuals). The characteristics of the dataset generated are found to depend on: branding and key features of the app, the subset of the population who engage, the subset that actively retain the app over time, the subset who engage in additional voluntary data collection, e.g. experience sampling, voluntarily offering personal data such as age and gender and voluntarily using features such as correction of mode where the app has mis-recorded. A computed Index of Multiple Deprivation highlights interfaces with established datasources and results show the distribution of transport-health outcomes from transport initiatives by sub-group.
\end{abstract}

Keywords: Track-and-Trace; equity, transport-health impacts, sustainable transport, smartphone 